Objective To assess the knowledge and practice of pulse oximetry screening for critical congenital heart defect in newborn infants by paediatricians in Benin.

Method This was a phone survey or a direct interview that interested pediatricians in Benin. The data examined included the seniority of paediatricians, their location, their knowledge and practice of pulse oximetry screening for critical congenital heart defect in newborn infants. The data has been analyzed with EPI info 3.5.4 software.

Results we have identified 84 paediatricians but only 55 $(65.47 \%)$ participated in this survey. The average duration of paediatric practice was $10.6 \pm 8.04$ years (1-29). Most of them exercised in LITTORAL district $(\mathrm{n}=34)$ and in private clientele $(n=22)$. Respondents reported hearing about pulse oximetry screening for critical congenital heart defect in newborn infants in $65.45 \%$ of cases. Only $12.73 \%$ were aware of a screening protocol and 16 paediatricians routinely practised it. Only one respondent had a program for screening of critical congenital heart defects by pulse oximetry at its center. The exercise time was inversely associated with knowledge of this screening $(p=0.005)$. This knowledge did not influence the practice of screening critical congenital heart defect in newborn infants $(\mathrm{p}=0.18)$.

Conclusion Pulse oximetry screening for critical congenital heart defect in newborn infants is few used by paediatricians in Benin. Its systematization would improve the screening.

\section{P437 HEALTHCARE PROVIDER'S PRACTICES REGARDING PAIN MANAGEMENT IN NEONATAL DEPARTMENTS}

Anastasiya Vanyarkina*, Alla Petrova, Lyubov Rychkova. Scientific Centre for Family Health and Human Reproduction Problems, Irkutsk, Russian Federation

\subsection{6/archdischild-2019-epa.773}

Pain control is one of the universal strategies for care delivery in all neonatal departments. The problem of pain management in neonates is of great importance for a medical institution, and not only the accountability of individual specialists.

The aim to analyze healthcare provider's awareness about pain management in newborns.

Methods Cross-sectional descriptive study was conducted at in neonatal departments of Perinatal Centre (III level), Irkutsk, Russia, from April to June 2018. Data were collected from 61 respondents: 46 (75.4\%) nurses and 15 (24.6\%) physicians. The ad hoc questionnaire contained 13 questions regarding respondents' demographics, professional qualification, their knowledge and practices in neonatal pain management. Part of the questionnaire contained information about availability and quality of local pain control guidelines in services. Pain intensity perception during painful procedures was described using a 5-point Likert scale. Data were analyzed using «Statistica 6.0» soft. The level of significance was $\mathrm{p}<0.05$.

Results Most respondents were female $(n=59)$, aged 23 to 60 years, and $56 \%$ had 5 to 10 -year experience in providing neonatal care.

The majority $(88.5 \%)$ always evaluated pain in their units using a validated EDIN scale. However 38 (62.2\%) respondents doubted the efficacy of this tool.

All professionals considered chest drainage $(p=1.0)$, lumbar puncture $(p=0.9)$, intramuscular injection $(p=1.0)$ to be the most painful procedures (4-5 - points Likert scale). Statistically significant differences among nurses and physicians were found between their perception of pain intensity during intravenous cannulation and heel pricks $(p=0.001$; $\mathrm{p}=0.009$, respectively). Physicians considered tracheal suctioning $(\mathrm{p}=0.02)$ to be the most painful procedure. All staff considered gastric tube insertion $(p=1.0)$ to be the procedure with minimal pain (1- points Likert scale). All professionals took pharmacological measures for pain relief during chest drainage $(p=0.8)$, tracheal intubation $(p=0.8)$, lumbar puncture $(\mathrm{p}=1.0)$. The most reported non-pharmacological measure for neonatal pain therapy was oral glucose both for nurses and physicians (84\% vs 75\%, respectively). Majority of nurses reported of non-pharmacological pain relief measures used for all routine painful procedures irrespective of pain intensity.

Conclusion The survey showed that the neonatal pain assessment is not systematic, pharmacological measures for pain management are used limitedly due to reservations about analgesic-related neurotoxicity, despite of pain intensity. Non-pharmacological methods are more used according to the staff, especially nurses, reports.

\section{P438 DESIGN OF AN ASSESSMENT TOOL TO EVALUATE NEONATAL CARE PRACTICE ACROSS A RURAL PROVINCE IN CAMBODIA AND SUBSEQUENT INITIATION OF A COMPREHENSIVE NEONATAL TRAINING PROGRAMME FOR MIDWIVES, NURSES AND DOCTORS}

${ }^{1}$ Ailbhe McGrath* ${ }^{,}{ }^{1}$ Nicola Smith, ${ }^{1}$ Manila Prak, ${ }^{1}$ Arthur Riedel, ${ }^{1}$ Neary Sopheak, ${ }^{1}$ Dary Vanna, ${ }^{1}$ Ele Baker, ${ }^{1}$ Isata Gborie, ${ }^{1}$ Lorn Leouk, ${ }^{1,2,3,4}$ Claudia Turner. ${ }^{1}$ Global Child Health Programme, Angkor Hospital for Children, Siem Reap, Cambodia; ${ }^{2}$ Cambodia Oxford Medical Research Unit, Siem Reap, Cambodia; ${ }^{3}$ Faculty of Tropical Medicine, Mahidol University, Bangkok, Thailand; ${ }^{4}$ Centre for Tropical Medicine and Global Health, Nuffield Department of Clinical Medicine, University of Oxford, Oxford, UK

\subsection{6/archdischild-2019-epa.774}

Introduction The neonatal mortality rate (NMR) in Cambodia is 18 per 1000 births. The NMR in rural areas is higher, with a reported rate of 25 per 1000 in the province being studied. A large-scale programme 'The Saving Babies Lives Programme' is currently being piloted to decrease neonatal deaths in Cambodia. An assessment of the current neonatal care practice was necessary to highlight areas for improvement. Our aim was to create an assessment tool to obtain a comprehensive overview of neonatal services to inform the implementation of a sustainable improvement strategy including a comprehensive training programme for midwives, nurses and doctors.

Methods An assessment tool was adapted from the KAP survey model (Knowledge, Attitudes and Practice). Two further sections were added; Equipment and Staffing, forming a 'KAPES survey'. Tablet-based questionnaires were created using KoboCollect Toolbox. Surveys were written in English and translated into Khmer. National and international guidelines and teaching materials were reviewed to decide core teaching topics and the level of care to be provided at each facility. The research team were trained as instructors and mentors.

Results Knowledge and attitude questions were developed across key domains depending on staff member (midwife, nurse or doctor) in the format of multiple choice questions (MCQs) and statements with a Likert scale. Practice, Equipment and Staffing (PES) included multiple question types and 
were adapted specific to location (health centre/hospital). Surveys were undertaken across 45 health centres and 1 hospital. Although knowledge rates were acceptable, attitudes continuously fell into indifferent or negative ranges in certain domains.

A multiple tiered neonatal training programme was developed, depending on staff type (midwife, nurse or doctor) comprising of a classroom-based residential component, shadowing at a tertiary neonatal facility and an extensive mentorship period. Teaching materials, assessments and logbooks were prepared, tailored to conditions commonly encountered and resources available locally, written in English and translated into Khmer. Where resources were deficient, plans to supply equipment were initiated. Development of a neonatal care unit was commenced.

Conclusions An assessment tool called a 'KAPES survey' was created to comprehensively assess neonatal services in rural Cambodia. A comprehensive multiple-tier neonatal training programme was developed and adapted to specific needs. Initial data guided implementation of the strategy to decrease the NMR. Repeating the survey will evaluate progress of the intervention. Ultimately, this tool and programme could be replicated across the country and may be useful in other middle to low-income countries.

\section{P439 A RETROSPECTIVE AUDIT LOOKING AT ECHO FINDINGS IN BABIES BORN WITH DOWN SYNDROME IN A TERTIARY NEONATAL UNIT}

Sarah Kyne*, Anna Curley. Neonatal Department, National Maternity Hospital, Holles Street, Dublin, Ireland

\subsection{6/archdischild-2019-epa.775}

Aims Down Syndrome (T21) is the most frequent chromosomal anomaly, occurring in one per 546 live births in Ireland ${ }^{1}$. Population cohort studies show cardiac anomalies are found in $57 \%$ of babies diagnosed with T21 with atrio-ventricular septal defects being the most common presentation ${ }^{2}$. Routine antenatal scans have meant that some babies with T21 are diagnosed with cardiac anomalies before birth. Our local guidelines emphasise that all children diagnosed with T21 have an early postnatal echocardiogram and referral to consultant cardiologist. Our aim was to assess whether we were meeting this objective and to describe the range of cardiac diagnoses in our cohort.

Methods Setting was a tertiary maternity hospital (8,000 birth per annum). This was a retrospective chart audit of all babies born 2016-2017 with a diagnosis of T21.

\begin{tabular}{ll} 
Abstract P439 Table 1 & \\
\hline Cardiac Anomaly & Number of Babies \\
\hline AVSD & 9 \\
ASD & 7 \\
Pulmonary hypertension & 7 \\
VSD & 6 \\
PDA & 6 \\
Normal Echo & 5 \\
\hline
\end{tabular}

Results 37/42 babies diagnosed with T21 were included in the study. 54\% of these babies needed admission to the baby unit. Mean age at birth was $35+6$ weeks, birth weight $2.9 \mathrm{~kg} .12$ babies were antenatally diagnosed. Cardiac findings at the 20week scan led to $67 \%$ of antenatal diagnoses. Of the four babies antenatally diagnosed with no cardiac findings at 20week scan; 1 had a small ASD on postnatal echo and the second had no cardiac findings. All babies, regardless of cardiac findings on clinical examination, received a postnatal echo. 36 received an echo while still an inpatient. $87 \%$ had at least one cardiac anomaly and $24 \%$ of the babies had an AVSD confirmed on echo. The table 1 below shows the top 7 (of 20) cardiac diagnoses from postnatal echo.

Conclusion One third of babies were diagnosed antenatally despite a high incidence of postnatal cardiac findings Postnatally $87 \%$ of babies had a cardiac anomaly with AVSD in $24 \%$. There is a wide variety of cardiac findings in this cohort of babies.

\section{REFERENCES}

1. Johnson Z, Lillis D, Delany V, et al. (1996) The epidemiology of Down syndrome in four counties in Ireland 1981-90. J Public Health Med 18:78-86

2. Weijerman ME, van Furth AM, Vonk Noordegraaf A, van Wouwe JP, Broers CJ, Gemke RJ. Prevalence, neonatal characteristics, and first-year mortality of Down syndrome: a national study. J Pediatr.2008;152(1):15-19

\section{P440 CADIAC NEONATAL LUPUS}

${ }^{1}$ Afef Ben Thabet, ${ }^{1}$ Mouna Loukil, ${ }^{1}$ Amel Ben Hamed, ${ }^{1}$ Chiraz Regaieg*, 'Manel Charfi, ${ }^{2}$ Mohamed Derbel, ${ }^{1}$ Nedia Hmida, ${ }^{1}$ Ridha Regaieg, ${ }^{3}$ Dorra Abid, ${ }^{2}$ Kais Chaabene, ${ }^{3}$ Samir Kamoun, ${ }^{1}$ Amira Bouraoui, ${ }^{1}$ Abdellatif Gargouri. 'Department of neonatology, Hedi Chaker Hospital, Sfax, Tunisia; ${ }^{2}$ Departement of Obstetrics and Gynecology, Hedi Chaker Hospital, Sfax, Tunisia; ${ }^{3}$ Cardiology Department , HediChaker Hospital, Sfax, Tunisia

\subsection{6/archdischild-2019-epa.776}

Background Neonatal lupus erythematosus (NLE) is an uncommon acquired autoimmune disease caused by transplacental passage of maternal anti-SSA/Ro and anti-SSB/La antibodies. The most common effects of NLE include cutaneous involvement and congenital heart block (CHB). The death rate of complete congenital heart block (CCHB), which is the most severe clinical manifestation, is as high as $20 \%$ to $30 \%$. That's why, early recognition of infants at risk is important.

Objectives To investigate the clinical features and long-term prognosis of cardiac NLE.

Methods A retrospective descriptive study of nine cases with cardiac events of neonatal lupus over a 15 -year period from January 2003 to December 2018. The diagnostic criteria required the presence of clinical symptoms and positive autoantibodies both in the mother and her child.

Results There were 9 patients (male: female ratio of 6:3). Sjögren syndrome was the most common maternal involvement. CCHB was detected in 7 patients of whom 2 patients had an antenatal diagnosis. Two other patients were presented with a cardiomyopathy with immediate heart failure in one case. Antenatal treatment with glucocorticoids and/or hydroxychloroquine was used in 3 cases (maternal connective disease in 2 cases and CCHB in 1 case). After birth, 2 patients were treated with isoprenaline and 6 of the 8 patients diagnosed with the $\mathrm{CHB}$ received single-chamber pacemaker implants. Immediate evolution was favorable for all patients. During the follow-up, two infants died. A cardiomyopathy appeared in 